\title{
Transverse acousto-electric effect in superconductors
}

\author{
P. Lipavskýa ${ }^{\text {J J Koláček }}{ }^{\mathrm{b}}$, P.-J. Lin ${ }^{\mathrm{c}}$ \\ ${ }^{a}$ Faculty of Mathematics and Physics, Charles University, Ke Karlovu 3, 12116 Prague 2, Czech Republic \\ ${ }^{b}$ Institute of Physics, Academy of Sciences, Cukrovarnická 10, 16200 Prague 6, Czech Republic \\ ${ }^{c}$ Research Department, Universal Analytics Inc., Airdrie, AB T4B 2A4, Canada
}

\begin{abstract}
We formulate a theory based on the time-dependent Ginzburg Landau (TDGL) theory and Newtonian vortex dynamics to study the transverse acousto-electric response of a type-II superconductor with Abrikosov vortex lattice. When exposed to a transverse acoustic wave, Cooper pairs emerge from the the moving atomic lattice and moving electrons. As in the Tolman-Stewart effect in a normal metal, an electromagnetic field is radiated from the superconductor. We adapt the equilibrium-based TDGL theory to this non-equilibrium system by using a floating condensation kernel. Due to the interaction between normal and superconducting components, the radiated electric field as a function of magnetic field attains a maximum value occurring below the upper critical magnetic field. This local increase in electric field has weak temperature dependence and is suppressed by the presence of impurities in the superconductor.
\end{abstract}

Keywords: superconductivity, acousto-electric effect, Abrikosov vortex, Tolman-Stewart effect PACS: 74.20.De, 74.25.Ld, 72.50.+b

\section{Introduction}

Superconductivity appears at low temperatures when materials are rigid and fragile so that for the majority of experiments made in cryostat it is not necessary to consider any motion of the crystal. The standard time-dependent Ginzburg-Landau theory (TDGL), which contains the assumption of local equilibrium, and is formulated in the laboratory frame, is a powerful tool to study phenomena in the vicinity of the superconducting-normal phase transition. To study gyroscopes [1], gravitational wave antennae [2] and the interaction of the superconducting condensate with strong sound waves $[3,4,5,6,7,8,9,10,11,12,13]$, where the atomic lattice is in motion, an extension to TDGL is needed to accommodate the dynamical system.

In the presence of a transverse acoustic wave, the condensate does not experience friction with the crystal and its imperfections as Cooper pairs do not scatter on the underlying crystal or its imperfections. The moving lattice, however, acts on the condensate by three nondissipative mechanisms: (i) Induction: The motion of ions creates an electric current which affects the electrons by

Email addresses: lipavsky@karlov.mff .cuni.cz http://fu.mff.cuni.cz/theory/lipavsky/ (P. Lipavský), kolacek@fzu.cz (J. Koláček), fareh.lin@gmail.com (P.-J. Lin)

Preprint submitted to Physica $C$ magnetic induction. (ii) Entrainment: A Cooper pair of zero momentum in a moving crystal has nonzero velocity with respect to the crystal. This effect is particularly strong in dirty superconductors, where the mass of the Cooper pair is strongly renormalized, $m^{*} \gg 2 m_{e}$. In the reference frame locally moving with the lattice, entrainment together with the fictitious force compose the inertial force causing the Tolman-Stewart effect. (iii) Deformation potentials: Deformations of the crystal lead to local changes of the chemical potential and material parameters which control superconductivity, for example its critical temperature.

Because of the induction the supercurrent tends to oppose the ionic current, but the compensation is not always complete. For example, in a steadily-rotating (but stationary) superconductor, currents near the surface are only partly compensated and the residual current produces a magnetic field known as the London moment [14]. In non-stationary (oscillating) systems the compensation is even less effective. In particular, under the influence of the ultrasound wave, the inertial motion of normal electrons as well as superconducting electrons leads to nonzero bulk currents via the Tolman-Stewart effect. The oscillating current radiates electromagnetic waves $[6,7]$ so that the superconductor exhibits nonzero acousto-electric effect.

October 15, 2018 
Theoretical analysis of the acousto-electric field in superconductors has been performed assuming the fully superconducting state with no normal current [7]; the normal electrons are 'clamped' to the lattice [4, 5, 11, 12]. Using this assumption, the TDGL theory of Verkin and Kulik [15] (originally developed for the case of steady rotation when normal currents are absent) can be used to study the acousto-electric field.

Experimental studies on the acousto-electric field of hole-type metal (niobium) and electron-type borocarbide $\left(\mathrm{Y}_{0.95} \mathrm{~Tb}_{0.05} \mathrm{Ni}_{2} \mathrm{~B}_{2} \mathrm{C}\right)$ show a $10 \%$ increase of the radiated electric field as the material transforms into the superconducting state (Fig. 2 in Ref. [7]). This small change shows that, at least in the vicinity of the transition temperature, the normal and the superconducting currents are comparable, which is incompatible with the assumption of stationary ('clamped') normal electrons. Our aim is to develop a theory which accounts for coexisting normal and superconducting currents.

Here we study theoretically the effect of a transverse sound wave on a superconducting system near the superconducting-normal phase-transition line, $B_{c 2}$; the transverse wave propagates along the $z$ axis, and oscillates in the $x$ direction. In the transverse wave the material experiences shear stress only, without compression. According to the experimental studies by Fil et al [7], the changes of potential and material parameters caused by shear deformations can be disregarded. To accommodate this non-equilibrium system, we use a modified version of TDGL theory based on a microscopic derivation [16].

In Sec. 2 we formulate the set of TDGL equations for the dynamical system with oscillating atomic lattice driven by an external transverse acoustic wave. Motion of normal electrons is allowed and this current treated in a self-consistent way, instead of assuming the normal electrons move together 'clamped' to the ions as in $[7,4]$. As a result, the normal current is driven by inertial forces as in the normal-state Tolman-Stewart effect. Details of our derivation based on the Boltzmann equation are given in Appendix A. In Sec. 3 using Newtonian dynamics, we analyze the acousto-electric effect in the mixed state. Vortex dynamics in a steady state is deduced from the force balance on vortices. Magnus, pinning and transverse forces are considered, along with friction forces from the atomic lattice and from normal electrons $[17,18]$. The effective forces acting on the superconducting electrons are identified from the extended TDGL equation in Sec. 2. Next we discuss the skin effect and the matching of the internal field and the radiated electromagnetic wave at the surface. Sec. 3 councludes with the resulting complete set of equations.
Sec. 4 contains numerical computations of the radiated electric field, using material parameters provided in [7].

\section{TDGL theory}

To study the electrons in an oscillating atomic lattice, it is advantageous to choose the moving background as the reference frame as Cooper pairs emerge from the moving electrons. Previous study [16] shows that to apply standard TDGL theory to a dynamical system, it is optimal to choose a condensation kernel floating with the background. Here we omit the details and write down the set of equations known as the floating-kernel time-dependent Ginzburg-Landau (FKTDGL) equations.

Relaxation of the Ginzburg-Landau (GL) order parameter $\psi$ in the dynamical system is described by the FK-TDGL equation

$$
\begin{aligned}
\frac{1}{2 m^{*}}\left(-i \hbar \nabla-e^{*} \mathbf{A}-\mathbf{P}\right)^{2} \psi & -\alpha \psi+\beta|\psi|^{2} \psi \\
& =\Gamma\left(\frac{\partial}{\partial t}-i \frac{2}{\hbar} \mu\right) \psi
\end{aligned}
$$

with the molecular field

$$
\mathbf{P}=\chi^{*} m^{*} \dot{\mathbf{u}}+m^{*} \mathbf{v}_{n} .
$$

The first term of the molecular field is due to the entrainment effect caused by motion of the ionic lattice with velocity $\dot{\mathbf{u}}$; the mass of a Cooper pair is $m^{*}=$ $m_{0} /\left(1+\chi^{*}\right)$, where $m_{0}=2 m_{e}$ is twice the electron mass $m_{e}$. The corresponding superconducting current is

$$
\mathbf{j}_{s}=\frac{e^{*}}{m^{*}} \operatorname{Re}\left[\bar{\psi}\left(-i \hbar \nabla-e^{*} \mathbf{A}-\mathbf{P}\right) \psi\right] .
$$

The velocity $\mathbf{v}_{s}$ of the condensate can be defined using $\mathbf{j}_{s}=e^{*}|\psi|^{2} \mathbf{v}_{s}=e n_{s} \mathbf{v}_{s}$. Because of the presence of the second term $m^{*} \mathbf{v}_{n}$ in $\mathbf{P}$, the operator $\left(1 / m^{*}\right)(-i \hbar \nabla-$ $\left.e^{*} \mathbf{A}-\mathbf{P}\right)$ gives the velocity of Cooper pairs with respect to normal electrons $\mathbf{v}_{n}$. The current generated by the moving ions is

$$
\mathbf{j}_{l}=-e n \dot{\mathbf{u}},
$$

where $\mathbf{u}$ is the ion displacement caused by the transverse sound wave.

In our treatment we relax the requirement of Sonin [4] that the electrons move with the same velocity $\dot{\mathbf{u}}$ as ions. Instead we assume that as with the TolmanStewart effect in normal conducting metals [19], the normal electrons lag behind ions and move with velocity $\mathbf{v}_{n}$. The normal current $\mathbf{j}_{n}=e n \mathbf{v}_{n}$ can be obtained 
from the Boltzmann equations, shown in Appendix A; the electric current is

$$
\mathbf{j}_{n}+\mathbf{j}_{l}=\frac{\sigma_{n}}{e}\left(\mathbf{F}^{\prime}-\frac{\tau}{1+i \tau \omega} \frac{e}{m} \mathbf{B} \times \mathbf{F}^{\prime}\right)-v \dot{\mathbf{u}} .
$$

The effective driving force

$$
\mathbf{F}^{\prime}=-e \frac{\partial \mathbf{A}}{\partial t}-\nabla \mu+e \dot{\mathbf{u}} \times \mathbf{B}-m_{e} \ddot{\mathbf{u}}
$$

includes the effective electric field (first and second terms), a part of the Lorentz force $\mathbf{F}_{L}=e\left(\mathbf{v}^{\prime}+\dot{\mathbf{u}}\right) \times \mathbf{B}$, where $\mathbf{v}^{\prime}$ is electron velocity relative to the lattice, and the inertial force. These terms can be understood in the reference frame moving with the lattice, where the third term enters the electric field via a Lorentz transformation. The relaxation time $\tau$ comes from the normal conductivity $\sigma_{n}$, from (A.33). The last term in relation (5) results from the diffusion of the transverse momentum [20]; this is similar to the mechanism causing the shear viscosity. Detail derivation of (5), analogous to Ohm's law, from the Boltzmann equation can be found in Appendix A.

From the continuity equation $\nabla \cdot \mathbf{j}=0$ we can obtain for the chemical potential

$$
\nabla^{2} \mu=\frac{e}{\sigma_{n}} \nabla \cdot \mathbf{j}_{s}+e \nabla \cdot(\dot{\mathbf{u}} \times \mathbf{B}),
$$

which is simplified by the transversality condition $\mathbf{q} \cdot \mathbf{u}=$ 0 for wave vector $\mathbf{q}$. The total force has zero divergence, so $\nabla \cdot \ddot{\mathbf{u}}=0$. We consider a system with homogeneous conductivity, $\nabla \sigma_{n}=0$.

The vector potential A can be obtained from the Maxwell equation

$$
\nabla^{2} \mathbf{A}=-\mu_{0}\left(\mathbf{j}_{s}+\mathbf{j}_{n}+\mathbf{j}_{l}\right)
$$

we use the Coulomb gauge $\nabla \cdot \mathbf{A}=0$. To obtain the radiated electromagnetic wave, we must evaluate skin vector potential and match internal and external fields. In Sec. 3.3, we will show that the skin effect is negligible if the wavelength of radiation is much larger than the skin depth.

We have a three-component system consisting of normal electrons, condensate, and electromagnetic field. Equations (1), (3), (5), (7) and (8) form a complete set of equations of motion. We are interested below in Sec. 3 a case that the transverse sound wave interacts with a superconductor in the mixed state. Here we compare our theory with the TDGL theory of Verkin and $\mathrm{Ku}$ lik $[20,15,4]$ referred as VK-TDGL.

To make the comparison, we rewrite our equations in terms of the relative velocities with respect to the atomic lattice, that is, the relative velocity of normal electrons as $\mathbf{v}_{n}^{\prime}=\mathbf{v}_{n}-\dot{\mathbf{u}}$ and the relative velocity of the condensate as $\mathbf{v}_{s}^{\prime}=\mathbf{v}_{s}-\dot{\mathbf{u}}$.

In this notation, the molecular field (2) is

$$
\mathbf{P}=m_{0} \dot{\mathbf{u}}+m^{*} \mathbf{v}_{n}^{\prime} .
$$

The first term is the fictitious force obtained by Verkin and Kulik [15]. The second term which is absent in [15] is a correction due to non-zero velocity of normal electrons with respect to the ionic lattice. From Ohm's law (5), (A.33) and (A.36), we can see that the relative velocity is proportional to mean free path $\ell$. In the dirty limit $\left(\ell \ll \xi_{0}\right) \mathbf{v}_{n}^{\prime} \rightarrow \mathbf{0}$, hence the second term in (9) can be ignored; our theory then reduces to VK-TDGL.

\section{Vortex dynamics}

Near the normal and superconducting phase transition, vortex motion is well described by TDGL theory. By solving the TDGL equation with the assumption of rigid Abrikosov vortex lattice, the TDGL equation can be represented in the form of force balance of Newtonian equations. Here we consider a superconductor which occupies $z<0$ with rigid Abrikosov vortices; each vortex has a fluxon $\Phi_{0}$ along the $z$-axis; the magnetic induction is $\mathbf{B}=(0,0, B), B>B_{c 1}$; thus the interspacing between vortices is $a \sim \sqrt{\Phi_{0} / B}$. The transverse acoustic wave propagates along the $z$-axis and oscillates in the $x$ direction; the atomic lattice deformation can be evaluated as the real part of the complex function $\mathbf{u} \equiv \exp (i \omega t) \cos (q z)(u, 0,0)$.

This physical system contains variables at microscopic scale, such as parameters describing motion of electrons, and variables at mesoscopic scale, such as the wavelength of the acoustic wave. The typical wavelength of the acoustic wave is $\sim 100 \mu \mathrm{m}$, and that of the radiation is of the order of a metre, while spacing between vortices is $\sim 100 \mathrm{~nm}$.

Since we are interested in phenomena at mesoscopic scale, we can average a microscopic field $f$ locally to produce a mesoscopic field $\langle f\rangle$, by writing

$$
\langle f\rangle(t, \mathbf{r}):=\left(B / \Phi_{0}\right) \int_{C_{\mathbf{r}}} \mathrm{d} x^{\prime} \mathrm{d} y^{\prime} f\left(t, \mathbf{r}^{\prime}\right)
$$

where the 2-D region $C_{\mathbf{r}}$ is the size and shape of an elementary cell, but with centroid $\mathbf{r}$ rather than being aligned with the lattice.

The acoustic wave acts on the superconductor in a similar manner as far-infrared (FIR) light; the condensate accelerates, so $\left\langle\mathbf{v}_{s}\right\rangle \neq 0$. Following the idea by Sonin [4], we will use the theory of vortex motion 
derived and experimentally tested for FIR response to study the interaction of acoustic waves with a superconductor in the mixed state.

\subsection{Balance of forces on the condensate}

Under the influence of the transverse acoustic wave, the effective force driving Cooper pairs into motion can be identified as the time derivative of the effective vector potential $\mathbf{A}_{e f f}=\mathbf{A}+\mathbf{P} / e^{*}$ in (1):

$$
\langle\mathbf{F}\rangle=-\frac{\partial}{\partial t}\left\langle e^{*} \mathbf{A}+\mathbf{P}\right\rangle .
$$

This averaged local force is balanced by

$$
\langle\mathbf{F}\rangle=-e^{*} \dot{\mathbf{u}}_{v} \times\langle\mathbf{B}\rangle+m^{*} \frac{\partial\left\langle\mathbf{v}_{s}\right\rangle}{\partial t},
$$

where $\mathbf{u}_{v}$ is the velocity of Abrikosov vortices. The first term comes from induction and by itself would comprise the Josephson relation [21]; the second term is essential in dynamical systems when $\left\langle\dot{\mathbf{v}}_{s}\right\rangle \neq 0$. The full (12) is known as the inertial Josephson relation (IJR) which can be obtained either from the standard TDGL theory [22, 23] or hydrodynamic theory [24]. Gutlyanskiǐ uses an identical equation (Eqn. (1) of [5]) which he calls the London equation because of its inertial term.

\subsection{Balance of forces on vortices}

In a dynamical situation, a vortex experiences a number of forces [25]. The simplest equation of motion for a vortex is through the compensation of the Lorentz force and the Bardeen-Stephen friction force due to dissipative scattering of quasi-particles in the vortex-core region. In reality, impurity of a sample complicates the dynamics of vortices; it leads to such things as vortex pinning on the mesoscopic scale, and modified relaxation times of particles on the microscopic scale.

Here we adopt a widely-used equation from [25]. Considering all the forces on a vortex of unit length in the oscillating lattice,

$$
\begin{aligned}
e n_{s}\left(\dot{\mathbf{u}}_{v}-\left\langle\mathbf{v}_{s}\right\rangle\right) \times \mathbf{z} & =\eta_{\text {lat }}\left(\dot{\mathbf{u}}_{v}-\dot{\mathbf{u}}\right) \\
& +\eta_{q p}\left(\dot{\mathbf{u}}_{v}-\left\langle\mathbf{v}_{n}\right\rangle\right) \\
& +\alpha_{L}\left(\mathbf{u}_{v}-\mathbf{u}\right) \\
& +\alpha_{K K}\left(\dot{\mathbf{u}}_{v}-\dot{\mathbf{u}}\right) \times \mathbf{z} \\
& -\alpha_{I}\left(\dot{\mathbf{u}}_{v}-\left\langle\mathbf{v}_{n}\right\rangle\right) \times \mathbf{z},
\end{aligned}
$$

where we have defined $\mathbf{z}=\left\langle\mathbf{B}_{\text {eff }}\right\rangle /\left\langle B_{\text {eff }}\right\rangle$. The left side contains the hydrodynamic Lorentz and Magnus forces. The Magnus force can be obtained from the TDGL equation with the assumption of a rigid Abrikosov lattice. The Lorentz force comes from the effective magnetic field $\left\langle\mathbf{B}_{e f f}\right\rangle=\nabla \times\left\langle\mathbf{A}_{e f f}\right\rangle$. In the linear response region $\mathbf{u}_{v}-\left\langle\mathbf{v}_{s}\right\rangle$ is small, therefore the effect of the acoustic wave on the magnetic field can be neglected, so $\left\langle\mathbf{B}_{\text {eff }}\right\rangle \approx\langle\mathbf{B}\rangle$ and we may write $\mathbf{z} \approx\langle\mathbf{B}\rangle /\langle B\rangle=\hat{\mathbf{z}}$.

The forces on the right side of (13) arise from a more detailed microscopic picture. The first two terms are frictional forces of vortex with the ionic lattice, and with normal electrons. The pinning force with the Labush parameter $\alpha_{L}$ is proportional to the relative displacement of the vortex from a pinning centre fixed to the ionic lattice. The transverse force of Kopnin and Kravtsov [17], due to scattering on impurities, has coefficient $\alpha_{K K}$. Finally, the interaction of the vortex with quasiparticles has the transverse component of Iordanskii type [18] with coefficient $\alpha_{I}$.

Rewriting (13) in terms of relative velocities $\dot{\mathbf{u}}_{v}^{\prime}, \mathbf{v}_{s}^{\prime}$ and $\mathbf{v}_{n}^{\prime}$, we can separate the force imposed by the normal current, writing

$$
\begin{aligned}
-e n_{s}\left\langle\mathbf{v}_{s}{ }^{\prime}\right\rangle \times \mathbf{z}= & \eta \dot{\mathbf{u}}_{v}{ }^{\prime}+\alpha_{L} \mathbf{u}_{v}{ }^{\prime}-\alpha_{M} \dot{\mathbf{u}}_{v}{ }^{\prime} \times \mathbf{z} \\
& -\eta_{q p}\left\langle\mathbf{v}_{n}^{\prime}\right\rangle+\alpha_{I}\left\langle\mathbf{v}_{n}^{\prime}\right\rangle \times \mathbf{z},
\end{aligned}
$$

where $\eta=\eta_{l a t}+\eta_{q p}$ is the total coefficient of friction, and $\alpha_{M}=e n_{s}-\alpha_{K K}+\alpha_{I}$ accounts for both corrections to the Magnus force.

In the limit $\mathbf{v}_{n}^{\prime} \rightarrow \mathbf{0}$, corresponding to the 'clamped' electron model, (14) coincides with the equation for forces on the vortex lattice used by Fil et al [7]. This limit is justified for dirty materials where $\tau \omega \ll 1$ in Fil's measurement $\left(\tau \sim 10^{-13} \mathrm{~s}\right.$ and frequency 55 $\mathrm{MHz}$ ). The theory developed here without restriction to this limiting case is valid for moderately pure materials and higher frequencies near sub-gap frequencies.

\subsection{Skin effect and the Maxwell equation}

The vector potential inside the superconductor contains a large contribution $\mathbf{A}^{0}$ from the Abrikosov vortex lattice which satisfies $\partial_{t}\left\langle\mathbf{A}^{0}\right\rangle=0$, and a timedependent perturbation which is the sum of the internal field with space dependence given by the acoustic wave $\left\langle\mathbf{A}^{\prime}\right\rangle=\mathbf{A}^{\prime} \exp (i \omega t) \cos (q z)$ and the skin field $\left\langle\mathbf{A}^{\prime \prime}\right\rangle=\mathbf{A}^{\prime \prime} \exp \left(i \omega t+z / \lambda_{s k}\right)$ where $\lambda_{s k}$ is the skin depth.

We solve for the surface fields by writing $\left\langle\mathbf{v}_{s}^{\prime}\right\rangle(t, \mathbf{r})=$ $\mathbf{v}_{s}^{\prime} \exp (i \omega t) \cos (q z)$ (similarly for $\mathbf{v}_{n}$ and other fields), shifting our notation so that from here onwards; $\mathbf{v}_{s}^{\prime}$ refers to the field at the surface at $t=0$.

The Maxwell equation (8) gives

$$
q^{2} \mathbf{A}^{\prime}=\mu_{0} e\left(n_{s} \mathbf{v}_{s}^{\prime}+i \omega n_{s} \mathbf{u}+n \mathbf{v}_{n}^{\prime}\right)
$$


$\mathbf{v}_{s}^{\prime}$ depends only on the oscillating transport current, as the strong static diamagnetic currents forming vortices average to zero over a cell.

At the surface, the matching of the internal electromagnetic wave $\mathbf{A}^{\prime}(t, \mathbf{r})+\mathbf{A}^{\prime \prime}(t, \mathbf{r})$ and the outgoing radiation $\mathbf{A}_{\text {out }} \exp (i \omega(t-z / c))$ yields two conditions. The first condition, obtained from the Maxwell equation $\mathbf{E}=-\partial_{t} \mathbf{A}$, is

$$
\mathbf{A}_{\text {out }}=\mathbf{A}^{\prime}+\mathbf{A}^{\prime \prime} \text {. }
$$

The second condition, obtained from $\mathbf{B}=\nabla \times \mathbf{A}$, is

$$
-i \frac{\omega}{c} \mathbf{A}_{\text {out }}=\frac{1}{\lambda_{s k}} \mathbf{A}^{\prime \prime} .
$$

In (17) we used that the rotation of the field is proportional to $\sin (q z)$ which vanishes at the surface. Solving for the radiated field from (16) and (17), we find

$$
\mathbf{A}_{\text {out }}=\frac{1}{1+i \frac{\omega}{c} \lambda_{s k}} \mathbf{A}^{\prime} .
$$

Since the wavelength of the radiation is $c / \omega \gg \lambda_{s k}$, we can approximate the radiated field by the internal one, so $\mathbf{A}_{\text {out }}=\mathbf{A}^{\prime}$.

\subsection{Equations for surface fields}

The ionic displacement $\mathbf{u}$ is known. The vortex displacement $\mathbf{u}_{v}^{\prime}$, the condensate velocity $\mathbf{v}_{s}^{\prime}$, the normal velocity $\mathbf{v}_{n}^{\prime}$, and the vector potential $\mathbf{A}^{\prime}$ are required. Here we rewrite equations in a convenient form.

The vortex displacement given by (14) at frequency $\omega$ is

$$
\begin{aligned}
e n_{s} \hat{\mathbf{z}} \times \mathbf{v}_{s}^{\prime}= & \left(i \omega \eta+\alpha_{L}\right) \mathbf{u}_{v}^{\prime}+i \omega \alpha_{M} \hat{\mathbf{z}} \times \mathbf{u}_{v}^{\prime} \\
& -\eta_{q p} \mathbf{v}_{n}^{\prime}-\alpha_{I} \hat{\mathbf{z}} \times \mathbf{v}_{n}^{\prime} .
\end{aligned}
$$

The condensate velocity is obtained from the IJR (12) with the force (11)

$2 e \mathbf{A}^{\prime}=-2 m \omega_{c} \hat{\mathbf{z}} \times\left(\mathbf{u}_{v}^{\prime}+\mathbf{u}\right)-2 i \omega m_{e} \mathbf{u}-m^{*}\left(\mathbf{v}_{s}^{\prime}+\mathbf{v}_{n}^{\prime}+i \omega \mathbf{u}\right)$,

where $\omega_{c}=e B / m$ is the cyclotron frequency.

The normal velocity is obtained from Ohm's law (5); the electric field in the force (6) is needed. Using the periodicity of the Abrikosov vortex lattice, we obtained $\langle\nabla \mu\rangle=0$ in Appendix B. Together with $\mathbf{B} \cdot \mathbf{u}=0$, the normal velocity is

$$
\begin{aligned}
m \mathbf{v}_{n}^{\prime} & =-\frac{i \tau \omega}{1+i \tau \omega}\left[e \mathbf{A}^{\prime}-\frac{\tau \omega_{c}}{1+i \tau \omega} \hat{\mathbf{z}} \times e \mathbf{A}^{\prime}+\right. \\
& +\left(i \omega m_{e}+m \omega_{c} \frac{\tau \omega_{c}}{1+i \tau \omega}+\frac{e v}{\sigma_{n}}\right) \mathbf{u} \\
& \left.+\left(m \omega_{c}+i \omega m_{e} \frac{\tau \omega_{c}}{1+i \tau \omega}\right) \hat{\mathbf{z}} \times \mathbf{u}\right] .
\end{aligned}
$$

We have considered radiation in response to a transverse acoustic wave; dependent on magnetic field, temperature and relaxation time, the radiation can now be evaluated by solving (15), (19)-(21). With $\mathbf{B}$ and $\mathbf{q}$ along the $z$ axis, all of $\mathbf{u}, \mathbf{u}_{v}^{\prime}, \mathbf{v}_{s}^{\prime}, \mathbf{v}_{n}^{\prime}$ and $\mathbf{A}^{\prime}$ have zero $z$-components. We have eight algebraic equations for $x$ and $y$ components of four unknown vectors.

Our model relaxes the assumption of VK-TDGL theory that normal electrons are stationary with respect to ions, but neither treatment takes into account effects of thermal fluctuations which are particularly strong at the superconducting and normal phase transition. The effect of thermal fluctuations can be included by the introduction of Langevin forces $\zeta(\mathbf{r}, t)$ in the left side of the FK-TDGL equation (1), which equation describes the dynamics of the order parameter. In this paper, we will next restrict our discussion to the case of conventional superconductors where the macroscopic fluctuations are negligible.

\section{Numerical predictions}

We study numerically the acousto-electric effect in the mixed state of a superconductor and in the region beyond the superconducting-normal phase transition line $B_{c 2}$. In the normal state, our theory reproduces the Tolman-Stewart effect. The distance to the phase transition line is defined as $\delta b=\left(B-B_{c 2}(T)\right) / B_{c 2}(0)$. The normal state corresponds to $\delta b>0$, the superconducting state to $\delta b<0$. Residual-resistance ratio (RRR) measured in the normal state is used to quantify the effect of imperfection of the atomic crystal. Here we focus on the case of niobium; necessary material parameters are taken from the measurement by Fil et al [7]. Parameters regarding the forces on vortices are specified in Appendix D.

We first discuss the $\delta b$ dependence of the radiated electric field $E_{\|}$, parallel to the atomic displacement due to the transverse acoustic wave incident perpendicular to the surface. The radiated electric field is normalized by its magnitude at $B=0$ and $T=0$, where all electrons are in the condensate. Shown in Fig. 1 is the radiation calculated from two different models, the FKTDGL theory and the VK-TDGL theory, for a sample with an RRR value of 62 at $T=0.75 T_{c}$; this corresponds to Fil's Fig. 3 of [7].

The overall radiation at $T=0.75 T_{c}$ is smaller then the radiation at $T=0$ and $B=0$; the radiation increases when entering superconducting state and saturates as $B \rightarrow 0$. As expected, FK-TDGL theory gives a non-zero $E_{\|}$in the normal state, consistent with the 

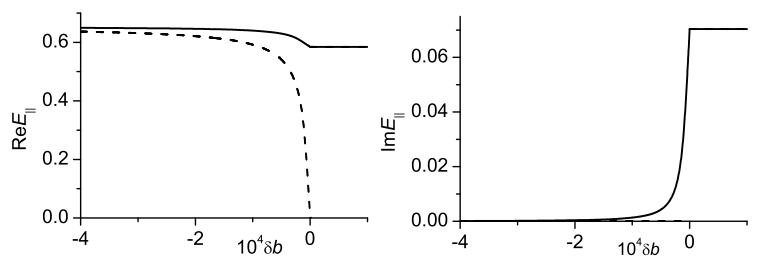

Figure 1: Parallel acousto-electric coefficient as function of magnetic field for superconductor with an RRR of 62: $E_{\|}$as a function of $\delta b$ near the critical line. The dashed line corresponds to the VK-TDGL model, and is not defined for positive $\delta b$. The solid line shows the FK-TDGL result, and is continuous in $\delta b$.
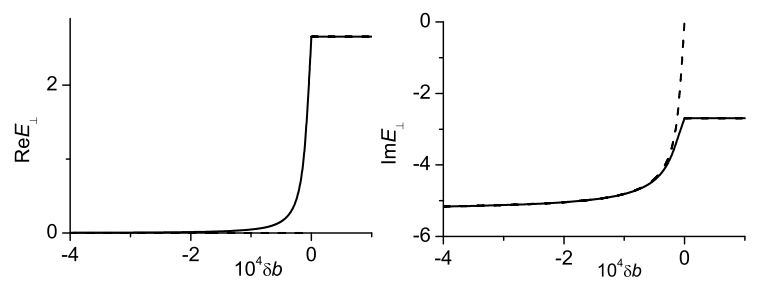

Figure 2: Transverse acousto-electric coefficient as a function of the $\delta b$ for RRR of 62: $E_{\perp}$, shown by the solid line, is continuous in FKTDGL theory while the dashed line shows a 'step' appearing in the VK-TDGL model.

Tolman- Stewart value, but VK-TDGL theory which ignores the effect of normal current gives $E_{\|}=0$. Nevertheless, both theoretical curves show marked changes near the phase transition, and the two curves coincide at small magnetic field where superconductivity is robust.

Both of these models suggest that $\operatorname{Im} E_{\|}$is negligible for very negative $\delta b$. Near the phase transition, the FKTDGL model indicates a fundamental increase when approaching $\delta b=0$ and then remains constant. The VK-TDGL curve shows that $\operatorname{Im} E_{\|}$remains negligible throughout the whole superconducting region.

$\operatorname{Re} E_{\perp}$ is dominated by the normal current and has similar behaviour to $\operatorname{Im} E_{\|}$. In Fig. $2, \operatorname{Re} E_{\perp}$ in the FK-TDGL model increases with $\delta b$ before the abrupt change near $\delta b=0$, while $\operatorname{Re} E_{\perp}$ remains negligible according to the VK-TDGL model. Im $E_{\perp}$ curves in the two models increase and coincide in the superconducting state; they separate when approaching $\delta b=0$. The VK-TDGL curve goes to zero at $\delta b=0$, while the FKTDGL curve shows a continuous change through transition into the normal state.

According to the FK-TDGL model, imperfections of a superconductor influence radiation near the phase
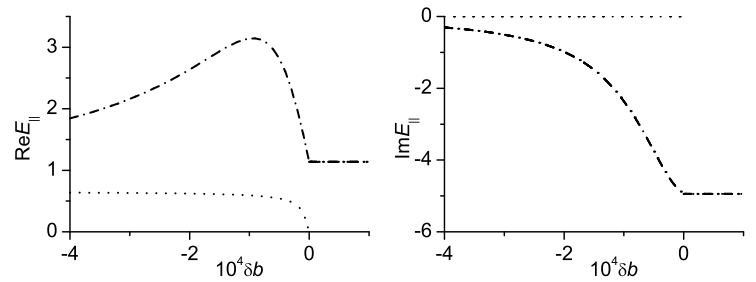

Figure 3: Parallel acousto-electric coefficient as a function of magnetic field for a superconductor with RRR of 620 at $T=0.75 T_{c}$ : The dot-dashed line shows the FK-TDGL result and the dotted line shows that of VK-TDGL; this convention is to facilitate comparison with the plots in Fig. 4.
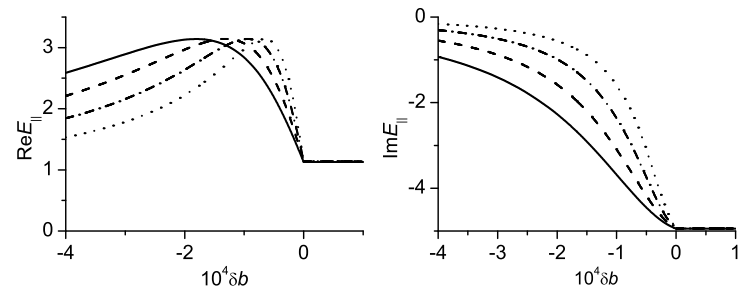

Figure 4: $E_{\|}$as a function of the $\delta b$ for RRR of 620: $t=0.1$ (solid line); $t=0.5$ (dashed line); $t=0.75$ (dot-dashed line); $t=0.99$ (dotted line).

transition. The FK-TDGL model suggests that a cleaner superconductor with RRR of 620 emits stronger radiation near the phase transition, shown in Fig. 3. The maximum $\operatorname{Re} E_{\|}$is around three times larger than its value in the normal state, or its value in the purelysuperconducting state at $T=0$ and $B=0$. The VKTDGL plot shows the radiated electric field increasing with $\delta b$ as in the dirtier superconductor shown in Fig. 1; the effect of impurities is negligible. The off-phase component $\operatorname{Im} E_{\perp}$ in Fig. 3 is suppressed in the superconducting state as in the dirtier superconductor discussed previously (with a different sign).

The enhancement of the radiation due to interaction between superconducting current and normal current is temperature dependent. $E_{\|}(\delta b)$ of the FK-TDGL model plotted at various temperatures is shown in Fig. 4. The location of the maximum gradually moves away from $\delta b=0$ as temperature decreases. While the peak is widened as temperature decreases, the magnitude of the peak changes little.

In contrast to the VK-TDGL model, FK-TDGL model accounts for the interaction between superconducting current and normal current. Our model provides 
a continuous description for a superconducting system in transition to normal state, and shows the TolmanStewart effect of normal metal in the normal state. When the superconducting system is away from the phase transition, the normal current contributes less and our model coincides with the VK-TDGL model. The VK-TDGL model is justified also in a dirty superconductor because the normal electrons scatter with impurities and thereby tend to move together with the lattice. However, in a clean superconductor the normal current contributes to the radiation; our model shows that radiation is enhanced due to the interaction between superconducting current and normal current. This enhancement occurs in superconducting state near the phase transition, and the field can reach three times that of radiation emitted in the normal state for certain values of $\delta b$.

\section{Summary}

The acousto-electric effect has been shown in the vicinity of the critical magnetic field to reveal the interference of the superconducting and the normal response. To investigate this interference, we have employed the time-dependent Ginzburg-Landau theory, taking into account the effect of the normal current on the formation of the condensate. This formulation with normal current had been derived earlier from the microscopic approach within the framework of a floating nucleation kernel.

The Ginzburg-Landau theory with the inertial term of Verkin and Kulik provides reliable predictions, save for within a very narrow vicinity of the critical line between the normal and the superconducting state. This deficiency is emphasized in cleaner samples. The interference appearing in this narrow vicinity shows enhancement which we expect to be observable, in particular in the case of Niobium with a high RRR.

\section{Acknowledgments}

This work was supported by MŠMT COST projects LD15062 and LD14060. P.-J. Lin acknowledges financial support from UA through SR-621-1207. Authors are grateful to V. D. Fil and D. V. Fil for many discussions on this subject and P. Matlock for critical reading.

\section{Appendix A. Transverse acousto-electric effect in normal metal}

In this appendix we derive the interaction of the transverse acoustic wave with the normal metal. The wave propagating in the $z$ direction, with wave vector $\mathbf{q}=$ $(0,0, q)$, is described by the amplitude of lattice deviation in the $x$-direction, $\mathbf{u}=(u, 0,0)$, with $u=u e^{i \omega t-i \mathbf{q} \cdot \mathbf{r}}$.

As the wave is transverse, the electric current generated by it is also transverse, therefore $\nabla \cdot \mathbf{j}=0$. From the equation of continuity follows that the charge density does not change $\partial_{t} \rho=-\nabla \cdot \mathbf{j}=0$ so that we can set $\phi=0$. We note that this argument holds to the linear order in $\mathbf{u}$. At quadratic order, there is a small charge transfer along the $z$ axis e.g. due to the Bernoulli effect; we neglect quadratic effects.

The generated transverse electric field is covered by the Maxwell equation $-\nabla^{2} \mathbf{A}=\mu_{0} \mathbf{j}$. Using $-\partial_{t} \mathbf{A}=\mathbf{E}$ yields

$$
q^{2} \mathbf{E}=-i \omega \mu_{0} \mathbf{j}
$$

We need to evaluate the current as a function of the electric field $\mathbf{E}$ and the deviation $\mathbf{u}$. To this end we use the Boltzmann equation in the relaxation time approximation

$$
\frac{\partial f}{\partial t}+\mathbf{v} \cdot \nabla f-\nabla \varepsilon \cdot \frac{\partial f}{\partial \mathbf{k}}=-\frac{1}{\tau} \delta f
$$

where $\delta f=f-\bar{f}$ is a deviation from local equilibrium. The local equilibrium distribution $\bar{f}$ represents electrons emitted from collisions with impurities and lattice vibrations. It has the same local density as the actual distribution

$$
2 \int \frac{\mathrm{d} \mathbf{k}}{(2 \pi)^{3}} \bar{f}=2 \int \frac{\mathrm{d} \mathbf{k}}{(2 \pi)^{3}} f=n,
$$

where the factor of two accounts for the sum over spins. Assuming isotropic collisions, the mean velocity of electrons emitted from collisions equals the velocity of the lattice,

$$
2 \int \frac{\mathrm{d} \mathbf{k}}{(2 \pi)^{3}} \bar{f} \mathbf{v}=n \dot{\mathbf{u}} .
$$

The quasiparticle energy in the lattice moving with velocity $\dot{\mathbf{u}}$ is

$$
\varepsilon=\frac{|\mathbf{k}-e \mathbf{A}|^{2}}{2 m_{e}}+\chi \frac{\left|\mathbf{k}-e \mathbf{A}-m_{e} \dot{\mathbf{u}}\right|^{2}}{2 m_{e}},
$$

where $\chi$ measures the renormalization of the inverse mass $1 / m=(1+\chi) / m_{e}$ in the normal state and the term proportional to it describes the normal entrainment. The corresponding quasiparticle velocity is

$$
\mathbf{v}=\frac{\partial \varepsilon}{\partial \mathbf{k}}=\frac{\mathbf{k}-e \mathbf{A}-\chi m \dot{\mathbf{u}}}{m} .
$$


The local equilibrium distribution $\bar{f}$ is centered around the mean momentum $\overline{\mathbf{k}}$,

$$
2 \int \frac{\mathrm{d} \mathbf{k}}{(2 \pi)^{3}} \bar{f} \mathbf{k}=n \overline{\mathbf{k}} .
$$

The condition (A.4) then gives

$$
\overline{\mathbf{k}}=e \mathbf{A}+m_{e} \dot{\mathbf{u}},
$$

where we have used $(1+\chi) m=m_{e}$. The local equilibrium is thus given by the Fermi-Dirac distribution

$$
\bar{f}(\mathbf{k}, \mathbf{r}, t)=f_{F D}(\bar{\varepsilon})
$$

with energy $\bar{\varepsilon}=|\mathbf{k}-\overline{\mathbf{k}}|^{2} / 2 m$ or

$$
\bar{\varepsilon}(\mathbf{k}, \mathbf{r}, t)=\frac{\left|\mathbf{k}-e \mathbf{A}(\mathbf{r}, t)-m_{e} \dot{\mathbf{u}}(\mathbf{r}, t)\right|^{2}}{2 m} .
$$

The total current is the sum of the ionic current -enu் and the electronic current

$$
\mathbf{j}=-e n \dot{\mathbf{u}}+2 e \int \frac{\mathrm{d} \mathbf{k}}{(2 \pi)^{3}} f \mathbf{v} .
$$

According to (A.4)

$$
2 e \int \frac{\mathrm{d} \mathbf{k}}{(2 \pi)^{3}} \bar{f} \mathbf{v}=e n \dot{\mathbf{u}}
$$

which exactly cancels the ionic current. The total current due to the deviation from local equilibrium is thus

$$
\mathbf{j}=2 e \int \frac{\mathrm{d} \mathbf{k}}{(2 \pi)^{3}} \delta f \mathbf{v} .
$$

The distribution $\delta f$ we will find from the Boltzmann equation (A.2)

$$
\left(\frac{1}{\tau}+\frac{\partial}{\partial t}+\mathbf{v} \cdot \nabla-\nabla \varepsilon \cdot \frac{\partial}{\partial \mathbf{k}}\right) \delta f=-\bar{I}
$$

with the source term

$$
\bar{I}=\frac{\partial \bar{f}}{\partial t}+\mathbf{v} \cdot \nabla \bar{f}-\frac{\partial \bar{f}}{\partial \mathbf{k}} \cdot \nabla \varepsilon .
$$

The local equilibrium depends on the time and space only via the central momentum $\overline{\mathbf{k}}$, therefore

$$
\begin{aligned}
\frac{\partial \bar{f}}{\partial t} & =\frac{\partial \bar{f}}{\partial k_{i}}\left(-e \frac{\partial A_{i}}{\partial t}-m_{e} \frac{\partial \dot{u}_{i}}{\partial t}\right), \\
\mathbf{v} \cdot \nabla \bar{f} & =\frac{\partial \bar{f}}{\partial k_{i}}\left(-e v_{j} \nabla_{j} A_{i}-m_{e} v_{j} \nabla_{j} \dot{u}_{i}\right), \\
-\frac{\partial \bar{f}}{\partial \mathbf{k}} \cdot \nabla \varepsilon & =\frac{\partial \bar{f}}{\partial k_{i}}\left(e v_{j} \nabla_{i} A_{j}+\left(m_{e}-m\right) v_{j}^{\prime} \nabla_{i} \dot{u}_{j}\right) .
\end{aligned}
$$

We have used the velocity relative to the lattice

$$
\mathbf{v}^{\prime}=\frac{\partial \bar{\epsilon}}{\partial \mathbf{k}}=\mathbf{v}-\dot{\mathbf{u}}
$$

Using relations $-\partial_{t} \mathbf{A}=\mathbf{E}, v_{j} \nabla_{i} A_{j}-v_{j} \nabla_{j} A_{i}=[\mathbf{v} \times \mathbf{B}]_{i}$ and $\partial_{t} \dot{\mathbf{u}}+\dot{\mathbf{u}} \cdot \nabla \dot{\mathbf{u}}=\ddot{\mathbf{u}}$, the source term can be expressed as

$$
\begin{aligned}
\bar{I} & =\frac{\partial \bar{f}}{\partial \mathbf{k}}\left(e \mathbf{E}+e \mathbf{v} \times \mathbf{B}-m_{e} \ddot{\mathbf{u}}+m_{e} \mathbf{v}^{\prime} \times[\nabla \times \dot{\mathbf{u}}]\right) \\
& -\frac{\partial \bar{f}}{\partial k_{i}} m v_{j}^{\prime} \nabla_{i} \dot{u}_{j} .
\end{aligned}
$$

As the local equilibrium distribution $\bar{f}$ depends only on $\bar{\varepsilon}$, the source term can be further simplified

$$
\bar{I}=\frac{\partial \bar{f}}{\partial \bar{\varepsilon}} \mathbf{v}^{\prime} \cdot\left(e \mathbf{E}+e \dot{\mathbf{u}} \times \mathbf{B}-m_{e} \ddot{\mathbf{u}}-m\left(\mathbf{v}^{\prime} \cdot \nabla\right) \dot{\mathbf{u}}\right),
$$

where we have used orthogonality $\mathbf{v}^{\prime} \cdot\left(\mathbf{v}^{\prime} \times \mathbf{B}\right)=0$ and $\mathbf{v}^{\prime} \cdot\left(\mathbf{v}^{\prime} \times[\nabla \times \dot{\mathbf{u}}]\right)=0$.

The current (A.13) in terms of the relative velocity (A.19) is

$$
\mathbf{j}=2 e \int \frac{\mathrm{d} \mathbf{k}}{(2 \pi)^{3}} \delta f \mathbf{v}^{\prime} .
$$

The term proportional to $\dot{\mathbf{u}}$ equals zero, because from (A.3) follows $\int d \mathbf{k} \delta f=0$.

To evaluate the deviation to terms linear in $\mathbf{u}$ we can neglect nonlinear terms in the left hand side of (A.14)

$$
\left(\frac{1}{\tau}+i \omega+\mathbf{v}^{\prime} \cdot \nabla-\nabla \bar{\varepsilon} \cdot \frac{\partial}{\partial \mathbf{k}}\right) \delta f=-\bar{I}
$$

The distribution $\delta f$ depends on $\mathbf{r}$ and $\mathbf{k}$ in two ways, via $\bar{\varepsilon}$ in $\bar{f}$, and via vectors $\mathbf{v}^{\prime}$ and $\nabla \bar{\varepsilon}$. Dependence on $\bar{\varepsilon}$ can be eliminated. Let us write the derivatives as

$$
\begin{aligned}
& \nabla \delta f=\frac{\partial \delta f}{\partial \bar{\varepsilon}} \nabla \bar{\varepsilon}+\left(\frac{\partial \delta f}{\partial \mathbf{r}}\right)_{\bar{\varepsilon}}, \\
& \frac{\partial \delta f}{\partial \mathbf{k}}=\frac{\partial \delta f}{\partial \bar{\varepsilon}} \mathbf{v}^{\prime}+\left(\frac{\partial \delta f}{\partial \mathbf{k}}\right)_{\bar{\varepsilon}} .
\end{aligned}
$$

The energy derivative cancels, therefore

$$
\left(\frac{1}{\tau}+i \omega\right) \delta f+\mathbf{v}^{\prime} \cdot\left(\frac{\partial \delta f}{\partial \mathbf{r}}\right)_{\bar{\varepsilon}}-\nabla \bar{\varepsilon} \cdot\left(\frac{\partial \delta f}{\partial \mathbf{k}}\right)_{\bar{\varepsilon}}=-\bar{I} .
$$

We will expand the solution in small $\tau /(1+i \tau \omega)$. The first order is

$$
\delta f_{1}=-\frac{\tau \bar{I}}{1+i \tau \omega}
$$


and the second order is

$$
\begin{aligned}
\delta f_{2}= & -\frac{\tau}{1+i \tau \omega}\left(\mathbf{v}^{\prime} \cdot\left(\frac{\partial \delta f_{1}}{\partial \mathbf{r}}\right)_{\bar{\varepsilon}}-\nabla \bar{\varepsilon} \cdot\left(\frac{\partial \delta f_{1}}{\partial \mathbf{k}}\right)_{\bar{\varepsilon}}\right) \\
= & \left(\frac{\tau}{1+i \tau \omega}\right)^{2}\left(\mathbf{v}^{\prime} \cdot\left(\frac{\partial \bar{I}}{\partial \mathbf{r}}\right)_{\bar{\varepsilon}}-\nabla \bar{\varepsilon} \cdot\left(\frac{\partial \bar{I}}{\partial \mathbf{k}}\right)_{\bar{\varepsilon}}\right) \\
= & \left(\frac{\tau}{1+i \tau \omega}\right)^{2} \frac{\partial \bar{f}}{\partial \bar{\varepsilon}} v_{j}^{\prime}\left(\nabla_{j}\left(v_{i}^{\prime} F_{i}^{\prime}-m v_{i}^{\prime} v_{k}^{\prime} \nabla_{k} \dot{u}_{i}\right)\right. \\
& \left.+e\left(\nabla_{l} A_{j}\right) \frac{\partial}{\partial k_{l}}\left(v_{i}^{\prime} F_{i}^{\prime}-m v_{i}^{\prime} v_{k}^{\prime} \nabla_{k} \dot{u}_{i}\right)\right)
\end{aligned}
$$

with the force

$$
\mathbf{F}^{\prime}=e \mathbf{E}+e \dot{\mathbf{u}} \times \mathbf{B}-m_{e} \ddot{\mathbf{u}} .
$$

In the linear response we can neglect $\dot{\mathbf{u}}$ in derivatives, $\nabla_{j} v_{i}^{\prime}=-(e / m) \nabla_{j} A_{i}$ and $\left(\partial v_{i}^{\prime} / \partial k_{j}\right)=(1 / m) \delta_{i j}$, therefore

$$
\begin{aligned}
\delta f_{2}= & \left(\frac{\tau}{1+i \tau \omega}\right)^{2} \frac{\partial \bar{f}}{\partial \bar{\varepsilon}}\left(\frac{e}{m} v_{j}^{\prime} F_{i}^{\prime}\left(\nabla_{i} A_{j}-\nabla_{j} A_{i}\right)\right. \\
& -e v_{j}^{\prime} v_{k}^{\prime}\left(\nabla_{i} A_{j}-\nabla_{j} A_{i}\right)\left(\nabla_{k} \dot{u}_{i}+\nabla_{i} \dot{u}_{k}\right) \\
& \left.-m v_{j}^{\prime} v_{i}^{\prime} v_{k}^{\prime} \nabla_{j} \nabla_{k} \dot{u}_{i}+v_{j}^{\prime} v_{i}^{\prime} \nabla_{j} F_{i}^{\prime}\right) \\
= & \left(\frac{\tau}{1+i \tau \omega}\right)^{2} \frac{\partial \bar{f}}{\partial \bar{\varepsilon}}\left(\frac{e}{m} \mathbf{v}^{\prime} \cdot\left[\mathbf{B} \times \mathbf{F}^{\prime}\right]\right. \\
& +m\left(\mathbf{v}^{\prime} \cdot \mathbf{q}\right)^{2}\left(\mathbf{v}^{\prime} \cdot \dot{\mathbf{u}}\right)-i\left(\mathbf{v}^{\prime} \cdot \mathbf{q}\right)\left(\mathbf{v}^{\prime} \cdot \mathbf{F}^{\prime}\right) \\
& \left.+i e \mathbf{B} \cdot\left(\left[\dot{\mathbf{u}} \times \mathbf{v}^{\prime}\right]\left(\mathbf{v}^{\prime} \cdot \mathbf{q}\right)+\left[\mathbf{q} \times \mathbf{v}^{\prime}\right]\left(\mathbf{v}^{\prime} \cdot \dot{\mathbf{u}}\right)\right)\right) .
\end{aligned}
$$

The function $\delta f=\delta f_{1}+\delta f_{2}$ includes terms odd and even in the velocity $\mathbf{v}^{\prime}$. We keep only the odd terms which contribute to the current,

$$
\begin{aligned}
& \delta f_{\text {odd }}=-\frac{\tau}{1+i \tau \omega} \frac{\partial \bar{f}}{\partial \bar{\varepsilon}} \mathbf{v}^{\prime} \cdot \mathbf{F}^{\prime} \\
& +\left(\frac{\tau}{1+i \tau \omega}\right)^{2} \frac{\partial \bar{f}}{\partial \bar{\varepsilon}}\left(\frac{e}{m} \mathbf{v}^{\prime} \cdot\left[\mathbf{B} \times \mathbf{F}^{\prime}\right]+m\left(\mathbf{v}^{\prime} \cdot \mathbf{q}\right)^{2}\left(\mathbf{v}^{\prime} \cdot \dot{\mathbf{u}}\right)\right) .
\end{aligned}
$$

The electric current is thus

$$
\mathbf{j}=\frac{\sigma_{n}}{e}\left(\mathbf{F}^{\prime}-\frac{\tau}{1+i \tau \omega} \frac{e}{m} \mathbf{B} \times \mathbf{F}^{\prime}\right)-v \dot{\mathbf{u}},
$$

where

$$
\sigma_{n}=-\frac{2 \tau e^{2}}{1+i \tau \omega} \frac{1}{3} \int \frac{\mathrm{d} \mathbf{k}}{(2 \pi)^{3}} \frac{\partial \bar{f}}{\partial \bar{\varepsilon}} v^{\prime 2}=\frac{\tau e^{2} n}{m(1+i \tau \omega)}
$$

is the usual conductivity in the absence of the magnetic field. The Hall component is implied by the force term $\mathbf{B} \times \mathbf{F}^{\prime}$.
The last term in (A.32) results from inhomogeneous velocity of the lattice, namely impurities and phonons. Its coefficient reminds the shear viscosity

$$
v=-\frac{2 \tau^{2} e m q^{2}}{(1+i \tau \omega)^{2}} \int \frac{\mathrm{d} \mathbf{k}}{(2 \pi)^{3}} \frac{\partial \bar{f}}{\partial \bar{\varepsilon}} v_{x}^{\prime 2} v_{z}^{\prime 2} .
$$

The integral over velocities in (A.34) in the zero temperature limit is

$$
\begin{aligned}
-2 & \int \frac{\mathrm{d} \mathbf{k}}{(2 \pi)^{3}} \frac{\partial \bar{f}}{\partial \bar{\varepsilon}} v_{x}^{\prime 2} v_{z}^{\prime 2} \\
= & \frac{2}{(2 \pi)^{3}} \int_{-1}^{1} d z z^{2}\left(1-z^{2}\right) \int_{-\pi}^{\pi} d \varphi \sin ^{2} \varphi \\
& \times \int_{0}^{\infty} d \bar{k} \delta\left(\bar{\varepsilon}-E_{F}\right) \frac{\bar{k}^{6}}{m^{4}} \\
= & \frac{1}{15 \pi^{2}} \frac{k_{F}^{5}}{m^{3}} \\
= & \frac{n v_{F}^{2}}{5 m},
\end{aligned}
$$

where we have used the density $n=k_{F}^{3} /\left(3 \pi^{2}\right)$ and the Fermi velocity $v_{F}=k_{F} / m$. Finally, we express the shear coefficient in terms of the mean free path $l=\tau v_{F}$

$$
v=\frac{e q^{2} n l^{2}}{5(1+i \tau \omega)^{2}} .
$$

For short lifetime $\tau \omega \rightarrow 0$, the coefficient $v$ agrees with the result of Fil [20].

\section{Appendix B. Chemical potential}

Here we show that the chemical potential can be excluded from assumptions dealing with the fields averaged over elementary cells of the Abrikosov vortex lattice.

Let us split the chemical potential as $\mu=\mu_{\mathbf{j}}+\mu_{\mathbf{u}}$, where the first term has the form standard in the TDGL theory

$$
\nabla^{2} \mu_{\mathbf{j}}=\frac{e}{\sigma_{n}} \nabla \cdot \mathbf{j}_{s}
$$

and the second term appears only in moving crystals and represents a change of the chemical potential due to the Lorentz force

$$
\nabla^{2} \mu_{\mathbf{u}}=e \nabla \cdot[\dot{\mathbf{u}} \times \mathbf{B}]
$$

Both potentials need boundary conditions which specify constant and linear terms. We use zero mean values, $\left\langle\mu_{\mathbf{j}}\right\rangle_{s}=0$ and $\left\langle\mu_{\mathbf{u}}\right\rangle_{s}=0$, where brackets denote average 
over sample volume. Since the system is periodic on the Abrikosov vortex lattice, this averaging is identical to averaging over single elementary cell and implies zero mean gradients $\left\langle\nabla \mu_{\mathbf{j}}\right\rangle=\mathbf{0}$ and $\left\langle\nabla \mu_{\mathbf{u}}\right\rangle=\mathbf{0}$.

It is necessary to show that the conditions $\left\langle\nabla \mu_{\mathbf{j}}\right\rangle=\mathbf{0}$ and $\left\langle\nabla \mu_{\mathbf{u}}\right\rangle=\mathbf{0}$ are not in conflict with equations (B.1) and (B.2) respectively. The source term in the right hand side of (B.1) is a sum of the transport supercurrent $\left\langle\mathbf{j}_{s}\right\rangle$ and the circulating current due to the Abrikosov vortex lattice. In the homogeneous Abrikosov lattice the transport supercurrent has zero divergence $\nabla \cdot\left\langle\mathbf{j}_{s}\right\rangle=0$ because of the translation invariance. The circulating component has zero divergence in the approximation of rigidly moving Abrikosov lattice. Beyond this approximation one finds contributions that are nonzero but periodic on the Abrikosov lattice giving the zero mean value, $\left\langle\nabla \cdot \mathbf{j}_{s}\right\rangle=0$. Zero mean value of the source term in (B.1) is not in conflict with the boundary condition $\left\langle\mu_{\mathbf{j}}\right\rangle=0$.

The source term in the right hand side of the equation (B.2) is rather complex. It simplifies in the linear approximation in $\mathbf{u}$ as

$$
\begin{aligned}
\nabla \cdot[\dot{\mathbf{u}} \times \mathbf{B}] & =-\dot{\mathbf{u}} \cdot[\nabla \times \mathbf{B}]+\mathbf{B} \cdot[\nabla \times \dot{\mathbf{u}}] \\
& =-\mu_{0} \dot{\mathbf{u}} \cdot\left(\mathbf{j}_{s}+\mathbf{j}_{n}+\mathbf{j}_{l}\right) \\
& \approx-\mu_{0} \dot{\mathbf{u}} \cdot \mathbf{j}_{s}^{0},
\end{aligned}
$$

where $\mathbf{j}_{s}^{0}$ is the supercurrent in the static Abrikosov lattice. We have used that the wave propagates along the magnetic field $\mathbf{B} \| \mathbf{q}$, therefore $\mathbf{B} \cdot[\nabla \times \dot{\mathbf{u}}]=0$. In the last step we have neglected terms beyond the linear response. Since there is no transport current in the static Abrikosov lattice $\left\langle\mathbf{j}_{s}^{0}\right\rangle=\mathbf{0}$, the source term has zero mean value, $\left\langle\dot{\mathbf{u}} \cdot \mathbf{j}_{s}^{0}\right\rangle=\dot{\mathbf{u}} \cdot\left\langle\mathbf{j}_{s}^{0}\right\rangle=0$. The boundary condition $\left\langle\mu_{\mathbf{u}}\right\rangle=0$ is thus not in conflict with the source term.

\section{Appendix C. Parameters of niobium}

First we list characteristic values. At the upper critical field at zero temperature $B=B_{c 2}=.49 \mathrm{~T}$, the cyclotron frequency $\omega_{c}=e B / m$ is $\omega_{c}=7.13 \cdot 10^{10} \mathrm{~s}^{-1}$, with the effective mass of niobium $m=1.2 m_{e}$. We note that niobium has a complicated Fermi surface appearing in the first, second and third Brillouin zone so that different effective masses appear, e.g. $m=3.2 m_{e}$ and $m=1.7 m_{e}$ from de Haas-van Alphen effect with and without phonon dressing [26]. In all cases the cyclotron frequency is much higher than the frequency of applied sound $\omega=2 \pi 5.5 \cdot 10^{7} \mathrm{~s}^{-1}$. The velocity of the transverse sound in niobium is $v_{s}=2100 \mathrm{~m} / \mathrm{s}$. This gives the wave vector $q=\omega / v_{s}=1.6 \cdot 10^{5} / \mathrm{m}$ and wave length $2 \pi / q=3.8 \cdot 10^{-5} \mathrm{~m}$.

Because of the complicated energy band structure, it is preferable to use characteristics of the Fermi surface rather than the effective mass and electron density. The single-spin density of states is $N_{0}=5.7 \cdot 10^{47} / \mathrm{Jm}^{3}$, and the average of the Fermi velocity over the niobium Fermi surface is $v_{F}=0.59 \cdot 10^{6} \mathrm{~m} / \mathrm{s}$, see Weber et al [27]. They enter the conductivity as $\sigma_{n}=\frac{2}{3} e^{2} N_{0} v_{F}^{2} \tau$.

The relaxation time $\tau$ depends on impurities. The niobium sample measured by Fil et al [7] reveals a step of acousto-electric effect in the zero magnetic field. Going from the superconducting state to the normal one, the magnitude reduces by $10 \%$ and the phase increases by $7^{\circ}$. Within the present theory it is reproduced by $\tau=1.2 \cdot 10^{-13} \mathrm{~s}$, which corresponds to the residual resistivity ratio $R R R=62$. This short relaxation time leads to rather small dimensionless numbers $\tau \omega_{c}=8.6 \cdot 10^{-3}$ and $\tau \omega=4.1 \cdot 10^{-5}$.

Different values one finds for pure samples. Weber et al [27] measured a sample of the residual resistivity ratio $R R R=2080$, giving the low temperature conductivity $\sigma_{n}=R R R / \rho_{n}=3 \cdot 10^{13} / \Omega \mathrm{m}$. This high conductivity corresponds to the relaxation time $\tau=8.9 \cdot 10^{-9} \mathrm{~s}$ with the dimensionless number $\tau \omega=3.1$. The mean number of circulations between collisions is $\tau \omega_{c}=635$. Since the relaxation time $\tau$ is proportional to the $R R R$, it is possible to prepare samples with $\tau$ from $10^{-8} \mathrm{~s}$ to $10^{-14} \mathrm{~s}$. Moreover, for a thin sample the magnetic field can be weak so that dimensionless numbers can have general values from small to values over unity.

The mean free path $l=v_{F} \tau$ spreads from $6 \cdot 10^{-9} \mathrm{~m}$ to $6 \cdot 10^{-3} \mathrm{~m}$. For the mean free path exceeding the wave length the theory of the normal acousto-electric effect is not fully justified because it is based on local approach with the lowest order nonlocal correction $v$. To stay in the region of validity we assume $\tau \ll 10^{-11}$ s for which $l \ll 1 / q$. The value $\mathrm{RRR}=620$ used for demonstration corresponds to $\tau=1.2 \cdot 10^{-12}$ s giving small dimensionless numbers $\tau \omega_{c}=8.6 \cdot 10^{-2}$ and $\tau \omega=4.1 \cdot 10^{-4}$.

Let us identify parameters for the superconducting state. For the dirty sample of Fil et al, the critical fields correspond to the GL parameter $\kappa=1.5$ given by the coherence length $\xi_{0}=2.6 \cdot 10^{-8} \mathrm{~m}$ and the London penetration depth $\lambda=3.9 \cdot 10^{-8} \mathrm{~m}$. Here $\lambda=\sqrt{m^{*} /\left(2 \mu_{0} e^{2} n_{s}\right)}$ with the Cooperon mass $m^{*}=2 m \hbar v_{F} /\left(\pi \Delta_{0} l_{\text {free }}\right)=$ $5.52 m_{e}$. We have used the BCS gap $\Delta_{0}=1.76 k_{B} T_{c}$. In the superconducting regime far from the critical temperature nonlocal contributions are negligible because $q \lambda=6.13 \cdot 10^{-3}$

Going to the clean limit there will be no dramatic 
changes. The Cooperon mass $m^{*}$ reaches the value of $2 \mathrm{~m}$. The London penetration depth decreases to $\lambda=2.3 \cdot 10^{-8} \mathrm{~m}$ and the GL parameter reduces close to the limiting value $\kappa \sim 1 / \sqrt{2}$. Samples of width comparable to the wave length $\sim 10^{-5} \mathrm{~m}$, but large in area $\sim 1 \mathrm{~cm}^{2}$, are penetrated by the magnetic field either in the form of Abrikosov vortices or in the form of slabs. We discuss the case in the vicinity of the critical temperature where sample becomes effectively thin as $\lambda$ is large so that vortices become preferable.

\section{Appendix D. Forces on vortex}

We take the friction according to Kopnin [25] (formula 12.38 with $B \rightarrow B_{c 2}$ limit of $\sigma_{f}$ given by formula 12.35) $\eta=\eta_{l a t}=\sigma_{n} B_{c 2}=1.8 \cdot 10^{6} \mathrm{C} / \mathrm{m}^{3}$. The quasiparticle friction is neglected in this approximation.

The coefficient of the Magnus-like force $\alpha_{M}=e n_{s}+$ $\alpha_{I}=3.5 \cdot 10^{9} \mathrm{C} / \mathrm{m}^{3}$ is dominated by the Iordanskii term $\alpha_{I}=e\left(n-n_{s}\right)$, see [25] formula below (14.97). We neglect the Kopnin-Kravtsov force.

The presented numerical results have been obtained with rather small Labusch coefficient $\alpha_{L} \ll e n_{s} / \omega$. We have found that acousto-electric effect remains the same within accuracy of figures even for values as large as $\alpha_{L} \sim 10^{3} e n_{s} / \omega$.

\section{References}

[1] E. T. Gawlinski, Phys. Rev. B 48 (1993) 351.

[2] A. Gulian, J. Foreman, V. Nikoghosyan, S. Nussinov, L. Sica, J. Tollaksen, Journal of Physics: Conference Series 507 (4) (2014) 042013.

[3] C. Hucho, M. Levy, Phys. Rev. Lett. 77 (1996) 1370-1373.

[4] E. B. Sonin, Phys. Rev. Lett. 76 (1996) 2794-2797.

[5] E. D. Gutlyanskiǐ, JETP Letters 67 (1998) 239.

[6] V. D. Fil‘, D. V. Fil, Y. A. Avramenko, A. L. Gaiduk, W. L. Johnson, Phys. Rev. B 71 (2005) 092504.

[7] V. D. Fil, D. V. Fil, A. N. Zholobenko, N. G. Burma, Y. A. Avramenko, J. D. Kim, S. M. Choi, S. I. Lee, Europhys. Lett. 76 (2006) 484.

[8] F. Jachmann, C. Hucho, Solid State Communications 142 (2007) 212-216.

[9] C. Hucho, F. Jachmann, Materials Science and Engineering: A 521-522 (2009) 307-309.

[10] C. Hucho, J. M. Carter, V. Müller, A. Petrean, W. K. Kwok, Physica C 332 (2000) 370-373.

[11] E. D. Gutlyanskiǐ, Phys. Rev. B 66 (2002) 052511.

[12] E. D. Gutlyanskiǐ, Physics of the Solid State 45 (2003) 812-815.

[13] J. Albert, E. M. Chudnovsky, Phys. Rev. B 77 (2008) 092506.

[14] F. London, Superfluids, Vol. 1, Wiley, New York, 1950.

[15] B. I. Verkin, I. O. Kulik, Sov. Phys. JETP 34 (1972) 1103.

[16] P. Lipavský, J. Bok, J. Koláček, Physica C: Superconductivity 492 (0) (2013) 144 - 151.

[17] N.-B. Kopnin, V. E. Kravtsov, JETP Lett. 23 (1976) 587.

[18] S.-V. Iordanskii, Sov. Phys. JETP 22 (1966) 160.

[19] T. J. Davis, G. I. Opat, Classical and Quantum Gravity 5 (7) (1988) 1011
[20] V. D. Fil, Low Temp. Phys. 27 (2001) 993.

[21] B. Josephson, Physics Letters 16 (3) (1965) 242 - 243

[22] P.-J. Lin, P. Lipavský, P. Matlock, Physics Letters A 376 (2012) 883-885.

[23] P. Matlock, Physica C 476 (2012) 59-62.

[24] A. Abrikosov, M. Kemoklidze, I. Khalatnikov, Sov. Phys. JETP 21 (1965) 506.

[25] N. B. Kopnin, Theory of Nonequilibrium Superconductivity, Claredon Press, Oxford, 2001.

[26] V. R. Karasik, I. Y. Shebalin, Soviet Physics JETP 30 (7) (1970) 1068-1075.

[27] H. W. Weber, E. Seidl, C. Laa, E. Schachinger, M. Prohammer, A. Junod, D. Eckert, Phys. Rev. B 44 (1991) 7585-7600. 